\author{
S. J. C. Doesborgh \\ W. M. E. van de Sandt- \\ Koenderman \\ D. W. J. Dippel \\ F. van Harskamp \\ P. J. Koudstaal \\ E. G. Visch-Brink
}

\section{Linguistic deficits in the acute phase of stroke}

Abstract Background and Purpose For the diagnosis of aphasia early after stroke, several screening tests are available to support clinical judgment. None of these tests enables the clinician to assess the underlying linguistic deficits, i. e. semantic, phonological and syntac-

Received: 12 September 2002

Received in revised form: 17 March 2003

Accepted: 28 March 2003

S. J. C. Doesborgh, MA · D. W. J. Dippel, MD, PhD - F. van Harskamp, MD · P. J. Koudstaal, MD, PhD · E. G. Visch-Brink, MA, PhD (ه)

Erasmus MC

Dept. of Neurology, Room Ee2291

P.O. BOX 1738

3000 DR Rotterdam, The Netherlands

Tel.: + 31-10/408-7817

Fax: + 31-10/408-9407

E-Mail: e.visch-brink@erasmusmc.nl

W. M. E. van de Sandt-Koenderman, MA

Rehabilitation Center Rijndam/

Aphasia Foundation Rotterdam

Rotterdam, The Netherlands tic deficits, which provides indispensable information for early therapeutic decisions. The ScreeLing was designed as a screening test to detect semantic, phonological and syntactic deficits. The ScreeLing's sensitivity, specificity and accuracy in detecting aphasia and semantic, phonological and syntactic deficits were determined. Methods The ScreeLing was validated in an acute stroke population against a combined reference diagnosis of aphasia (aphasia according to at least two of the following measures: neurologist's judgment, linguist's judgment, Tokentestscore). The three ScreeLing subtests were validated in the aphasic population against the presence or absence of a semantic, phonological and/or syntactic deficit according to an experienced clinical linguist. Results From a consecutive series of 215 stroke patients, 63 patients were included. The ScreeLing was an accurate test for the detection of aphasia (0.92), with a sensitivity of $86 \%$ and specificity of $96 \%$. Sensitivity of subtests was $62 \%$ for semantics, $54 \%$ for phonology and $42 \%$ for syntax. Specificity was $100 \%$ for semantics and phonology and $80 \%$ for syntax, and accuracy 0.84 for semantics, 0.87 for phonology and 0.64 for syntax. Conclusions The ScreeLing is an accurate test that can be easily administered and scored to detect aphasia in the first weeks after stroke. Furthermore, the ScreeLing is suitable for revealing underlying linguistic deficits, especially semantic and phonological deficits.

Key words aphasia $\cdot$ diagnosis . cerebrovascular accident $\cdot$ bedside tests $\cdot$ linguistic test

\section{Appendix}

\section{ScreeLing}

The ScreeLing is designed to take about 15 minutes to administer and has a simple right-or-wrong scoring system. The test consists of 3 subtests, each with four tasks:

\section{Semantics (24 items)}

Sem 1: word-picture matching (oral and written) with distracters from the same semantic field
Sem 2: judgment of anomalous and non-anomalous sentences

Sem 3: verbal semantic association

Sem 4: choosing odd-word-out

\section{Phonology (24 items)}

Phon 1: repetition of words with an increasing phonological complexity and word length

Phon 2: reading aloud of words with an increasing phonological complexity and word length

Phon 3: reading backwards of short words

Phon 4: auditory lexical decision 


\section{Syntax (24 items)}

Syn 1: sentence-picture matching

Syn 2: judgment of syntactic correctness

Syn 3: selecting the syntactically correct sentence

Syn 4: repetition of sentences consisting of mainly function words

\section{Introduction}

There are a number of short aphasia-screening tests that can be used to support clinical judgment, e.g. the Frenchay Aphasia Screening Test (FAST $[1,8]$ ), the Acute Aphasia Screening Protocol (AASP [4]) and the Ullevaal Aphasia Screening test (UAS test [15]). These tests are suitable for use in the hospital: they are short (5-15 minutes) and do not require extensive test material at the bedside. The screening tests are developed for determining the presence of aphasia, but often more detailed information is required. For shorthand communication with colleagues, it may be useful to specify aphasia type (e.g. Wernicke, Broca). However, there is growing evidence that a diagnosis in terms of affected linguistic levels - semantics (word meaning), phonology (word sound), syntax (grammatical structure) - is more useful than aphasia type [9]. A linguistic profile of the patient is needed for adequate referral and for guiding aphasia therapy: the practice standard for the treatment of patients with aphasia following left hemisphere stroke is cognitive linguistic therapy [3], i. e. therapy focused on the affected linguistic level(s). Treatment aimed at restoration of function, as in cognitive linguistic therapy, is argued to be especially appropriate in early acute stages, in order to converge with neural recovery [5]. In addition, it is important that patients and their families are informed about the linguistic pattern of the disorder in an early stage. This information can help them to develop coping strategies in communicative situations as soon as possible.

The ScreeLing [20] was developed as a screening test to measure impairment at the semantic, phonological and/or syntactic level in 15 minutes. In this study, the sensitivity and specificity of the ScreeLing for diagnosing aphasia and linguistic deficits in patients with acute stroke were estimated. The presence and severity of linguistic deficits were described for the patients individually and differences between subtests were computed for the patients as a group.

\section{Methods}

\section{Subjects}

Patients were recruited from the Rotterdam Stroke Databank, a prospective registry of patients with transient ischemic attack, ischemic stroke or primary intracerebral hemorrhage who are admitted to the Department of Neurology of the Erasmus Medical Center. All patients who were admitted between 1 August 2000 and $1 \mathrm{Au}-$ gust 2001 were considered for examination.

Native Dutch speakers who could be assessed between 2 and 11 days post stroke were included. Patients whose symptoms had subsided should have neurological signs on examination, to be included in the study. Patients who were illiterate, mentally retarded or premorbidly demented were excluded, as well as patients who had severe visual or auditory problems.

For the analysis of aphasic patients' subtest-scores, additional stroke patients with aphasia were recruited from a second hospital, the Medical Center Rijnmond Zuid, according to the same inclusioncriteria.

The study was approved of by the local Medical Ethics Committee. All patients gave verbal informed consent prior to their inclusion in the study.

\section{Materials and Methods}

The ScreeLing consists of three subtests - Semantics, Phonology and Syntax - of 24 items each (see appendix). Its sensitivity and specificity in detecting aphasia were determined by comparing the ScreeLing overall-score with a combined reference diagnosis. The reference diagnosis consisted of three measures, as we considered this to result in a more reliable and valid diagnosis than one that is based on only one measure. Patients were labeled with the reference diagnosis of 'aphasia' if they had aphasia according to at least two of the following measures: a) the 36-item version of the Tokentest [6], an often used and well-validated measure to assess the presence of aphasia, b) the independent judgment of a neurologist (based on clinical examination), c) the independent judgment of a linguist (based on an interview). The linguist and neurologist were blinded to the test results and to each other's judgment. The examiner who assessed the patients with the ScreeLing and the Tokentest, was blind to the judgment of the linguist and neurologist. The ScreeLing was always administered first; the examiner was therefore blind to the Tokentest-score.

The sensitivity and specificity of each ScreeLing subtest (Semantics, Phonology and Syntax) were determined by comparing the subtest-scores of patients with aphasia with the reference diagnosis: the judgment of an experienced linguist. Based on clinical linguistic assessment (not using tasks that were included in the ScreeLing), the linguist decided whether or not the patient had a semantic, phonological and/or syntactic deficit.

\section{Analysis}

Agreement within the combined reference diagnosis of aphasia was computed. A global assessment of the performance (or diagnostic accuracy) of the ScreeLing and its subtests was given by the area under the Receiver Operating Characteristic (ROC) curve. Sensitivity and specificity at the optimal cut-off point (i. e. maximizing the sum of the sensitivity and specificity) were determined. Differences between mean subtest scores were computed by means of paired samples ttests.

\section{Results}

A consecutive series of 215 patients was recruited from the Rotterdam Stroke Databank (Fig. 1). Of these patients, 111 were excluded from the present study, 56 $(51 \%)$ of whom were not available for testing between 2-11 days post onset owing to late admission, early discharge or death, $22(20 \%)$ were non-native speakers, 11 


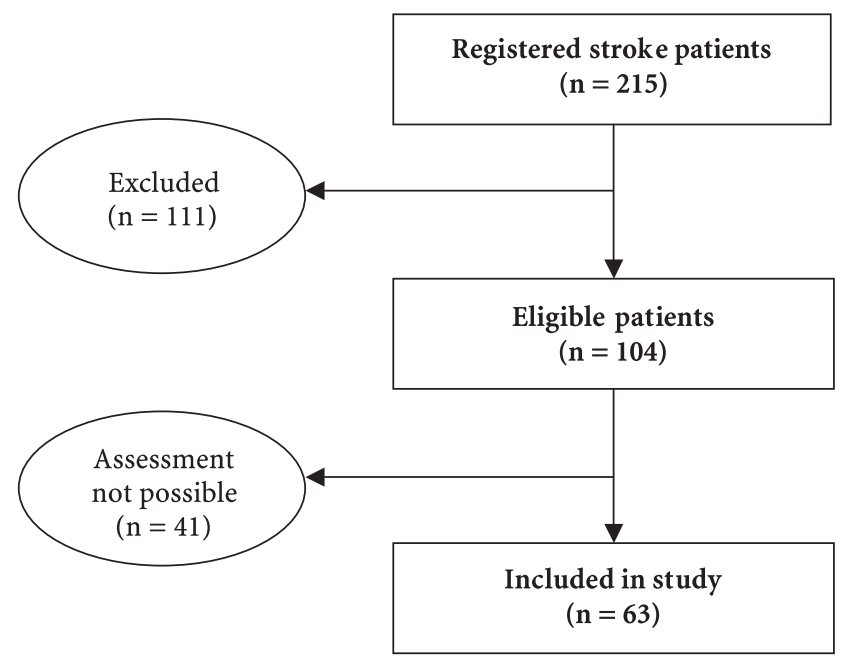

Fig. 1 Flowchart of inclusion of patients ${ }^{\mathrm{a}}$

a For the subtest analyses, another four patients were recruited from Medical Center Rijnmond Zuid

(10\%) had a TIA without neurological signs on admission, $10(9 \%)$ had premorbid visual or auditory problems, $6(5 \%)$ refused and $6(5 \%)$ were excluded for other reasons (dementia, mental retardation etc.). One-hundred-and-four patients were included, but $41(39 \%)$ of them could not be assessed owing to severe illness (32 patients) or visual-spatial problems (7 patients) or confusion secondary to stroke (2 patients).

A total of 63 patients was assessed. 43 patients were male and 20 were female. Mean age was 62 years (SD 16). Most patients $(86 \%)$ had an infarction. Forty-six percent of the patients had a left hemisphere stroke, $43 \%$ a right hemisphere stroke and $11 \%$ a brainstem or cerebellar stroke (Table 1).

There was high agreement between the neurologist and linguist (kappa $=0.84, \mathrm{p}<0.01$ ) between the Tokentest and the linguist $(\mathrm{kappa}=0.86, \mathrm{p}<0.01)$ and between the Tokentest and the neurologist (kappa $=0.77$, $\mathrm{p}<0.01)$.

Table 1 Baseline characteristics $(n=63)$

\begin{tabular}{llcll}
\hline & & $\begin{array}{c}\text { aphasia } \\
(\mathrm{n}=14)\end{array}$ & $\begin{array}{l}\text { no aphasia } \\
(\mathrm{n}=49)\end{array}$ & $\begin{array}{l}\text { whole group } \\
(\mathrm{n}=63)\end{array}$ \\
\hline age & Mean (sd) & $68(11)$ & $60(17)$ & $62(16)$ \\
sex & Male & $9(64 \%)$ & $34(69 \%)$ & $43(68 \%)$ \\
lesion-site & Left hemisphere & $13(93 \%)$ & $16(33 \%)$ & $29(46 \%)$ \\
& Right hemisphere & $1(7 \%)$ & $26(53 \%)$ & $27(43 \%)$ \\
& Cerebellum/brainstem & & $7(14 \%)$ & $7(11 \%)$ \\
stroke type & Hemorrhage & $2(14 \%)$ & $7(14 \%)$ & $9(14 \%)$ \\
& Infarction & $12(86 \%)$ & $42(86 \%)$ & $54(86 \%)$ \\
\hline
\end{tabular}

\section{ScreeLing overall-score $(n=63)$}

The median ScreeLing overall-score was 70 out of 72 . The optimal cut-off score was 65: patients were classified as aphasic if they scored 65 or less. This resulted in a sensitivity of $86 \%$ and a specificity of $96 \%$. The area under the ROC-curve (0.92) indicates that the ScreeLing was an accurate test (Fig. 2).

\section{Linguistic levels $(n=17)$}

Fourteen patients recruited from Erasmus MC had aphasia according to the reference diagnosis, i. e. by the Tokentest, the judgment of the neurologist and/or the judgment of the linguist (at least two of these measures). Of one of these patients, the linguistic level reference diagnoses were missing. The other aphasic patients were included into the subtest analyses. Furthermore, four patients with reference diagnosis aphasia were recruited from a second hospital, the Medical Center Rijnmond Zuid. Three patients were female and one patient was male. All had a left hemisphere infarction and mean age was 65 years (SD 17).

Semantics appeared to be the most sensitive, with a sensitivity of $62 \%$ at the optimal cut-off score, as compared with $54 \%$ and $42 \%$ for Phonology and Syntax. Specificity was $100 \%$ for all subtests. Accuracy was high for Semantics and Phonology, as indicated by the area under the ROC-curves (0.84 and 0.87) (Fig. 3), but for Syntax, the area under the ROC-curve was 0.64 (Table 2). Five patients $(29 \%)$ had a selective deficit: three patients

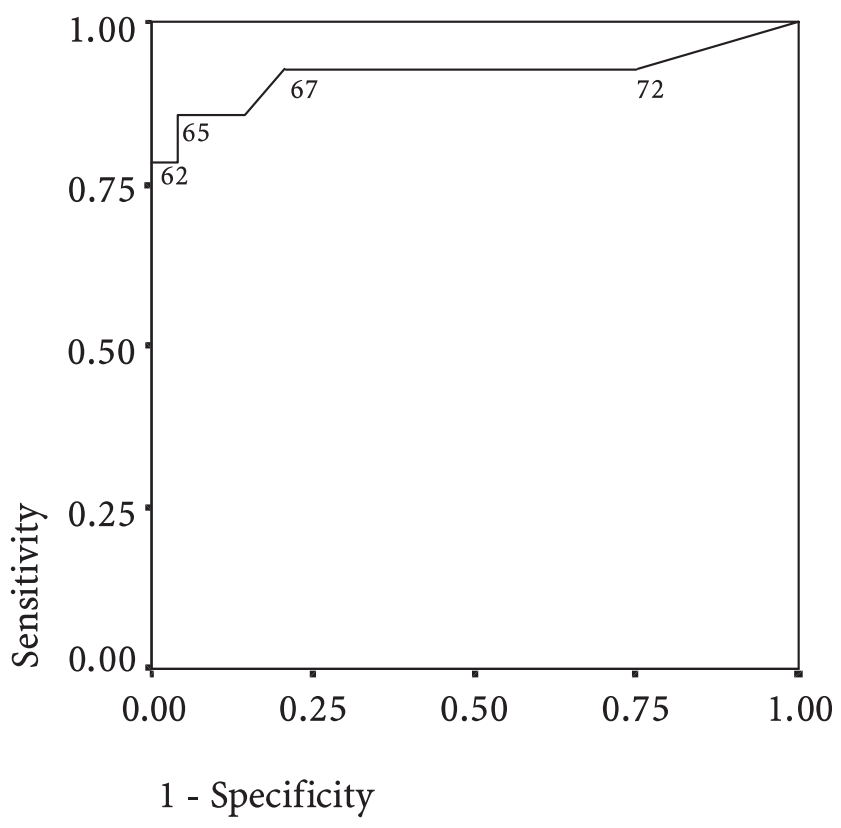

Fig. 2 ROC curve of ScreeLing overall score $(n=63)$ 

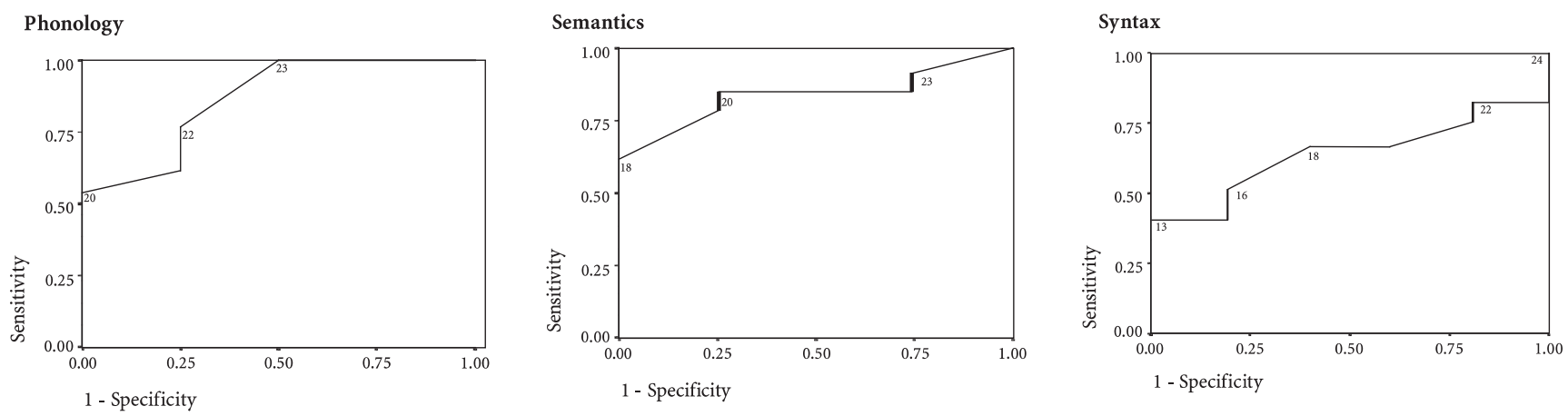

Fig. 3 ROC curves of ScreeLing subtests $(n=17)$

Table 2 Sensitivity, specificity and accuracy of ScreeLing subtests in 17 patients with aphasia

\begin{tabular}{llllllll}
\hline & $\begin{array}{l}\text { No } \\
\text { with } \\
\text { deficit }\end{array}$ & $\begin{array}{l}\text { No } \\
\text { without } \\
\text { deficit }\end{array}$ & $\begin{array}{l}\text { median } \\
\text { score (sd) }\end{array}$ & $\begin{array}{l}\text { Optimal } \\
\text { cut-off } \\
\text { point }\end{array}$ & Sensitivity & Specificity & Accuracy \\
\hline $\begin{array}{l}\text { Semantics } \\
(\max 24)\end{array}$ & 13 & 4 & $19(4.9)$ & 18 & $62 \%$ & $100 \%$ & 0.84 \\
$\begin{array}{l}\text { Phonology } \\
(\max 24)\end{array}$ & 13 & 4 & $21(4.5)$ & 20 & $54 \%$ & $100 \%$ & 0.87 \\
$\begin{array}{l}\text { Syntax } \\
(\max 24)\end{array}$ & 12 & 5 & $18(5.4)$ & 13 & $42 \%$ & $100 \%$ & 0.64 \\
\hline
\end{tabular}

scored below cut-off on Semantics (cases 8-10) and two on Phonology (cases 6 and 7) (Table 3).

Overall, the mean Phonology score was significantly

Table 3 ScreeLing subtest scores in 17 patients with aphasia

\begin{tabular}{llll}
\hline PT nr. & $\begin{array}{l}\text { Semantics } \\
\text { (cut-off: 18) }\end{array}$ & $\begin{array}{l}\text { Phonology } \\
\text { (cut-off: 20) }\end{array}$ & $\begin{array}{l}\text { Syntax } \\
\text { (cut-off: 13) }\end{array}$ \\
\hline 1 & $\mathbf{1 3}$ & $\mathbf{1 8}$ & $\mathbf{9}$ \\
2 & $\mathbf{8}$ & $\mathbf{1 9}$ & $\mathbf{9}$ \\
3 & $\mathbf{1 2}$ & $\mathbf{8}$ & $\mathbf{9}$ \\
4 & $\mathbf{9}$ & $\mathbf{1 2}$ & $\mathbf{9}$ \\
5 & $\mathbf{1 5}$ & $\mathbf{1 8}$ & $\mathbf{1 2}$ \\
6 & $21^{*}$ & $\mathbf{1 5}$ & $14^{*}$ \\
7 & $19^{*}$ & $\mathbf{2 0}$ & $18^{*}$ \\
8 & $\mathbf{1 7}$ & $21^{*}$ & 18 \\
9 & $\mathbf{1 8}$ & 22 & 15 \\
10 & $\mathbf{1 6}$ & 23 & 20 \\
11 & 23 & 23 & 21 \\
12 & $22^{*}$ & $24^{*}$ & $23^{*}$ \\
13 & $24^{*}$ & 23 & 24 \\
14 & 19 & 21 & $20^{*}$ \\
15 & 19 & 22 & 18 \\
16 & 20 & $23^{*}$ & $19^{*}$ \\
17 & 24 & $24^{*}$ & 24 \\
\hline
\end{tabular}

Patient-numbers in italics refer to patients from Medical Center Rijnmond Zuid; values in bold are equal to or below cut-off.

${ }^{*}$ reference diagnosis (judgment linguist) $=$ no deficit at this linguistic level higher than the mean Semantics score (mean difference $=2.17,95 \%$ C. I. $=0.28 ; 4.05)$ and the mean Syntax score $($ mean difference $=3.17,95 \%$ C. I. $=1.16 ; 4.72$ ).

\section{Discussion}

In this study, we found that the ScreeLing is an accurate test to detect aphasia between 2 and 11 days after stroke. The ScreeLing, which examines three linguistic levels, is very sensitive and specific and even exceeds aphasia screening tests that are based on a more general approach (FAST [1], UAS test [15]). Only two patients (4\%) who failed on the ScreeLing had a reference diagnosis 'no aphasia'. These patients were 4 and 8 days post onset, thus within our group of patients (mean number of days post onset: 5) they were not the patients who were most acute and supposedly most influenced by post stroke confounding variables. Two patients (14\%) had a ScreeLing-score above cut-off (65/72), whereas their reference diagnosis was aphasia. According to the linguist and the neurologist, these patients suffered from mild aphasia. This could not be detected by the ScreeLing, nor with the Tokentest, one of the most reliable tools for diagnosing aphasia $[2,7,17]$. In order to detect mild aphasia, observations of hesitations, self-corrections and performance in stressful situations (e. g. speeded naming) should be taken into account. These aspects were not included in the ScreeLing, because screening tests require unequivocal tasks that are easy to administer 
and score. Furthermore, there may not be an indication for disorder-oriented language therapy for patients with deficits that remain undetected after formal testing; more research on the recovery pattern of mild aphasia is needed.

Aphasia screening tests are applicable for only a limited proportion of acute stroke patients, mainly because many patients either die or recover early. Of the eligible stroke-patients, only $61 \%$ had the mental and physical strength to participate in the assessment in our study, despite the fact that the ScreeLing takes only 15 minutes to administer. Laska et al. [11] could assess a larger percentage of their acute stroke patients $(90 \%)$. Perhaps their inclusion was more selective (inclusion criteria were not reported), or less severe patients were admitted to their hospital.

The ScreeLing not only is an accurate test for detecting aphasia, but also provides information on the patients' linguistic abilities. The tests for assessing deficits at the linguistic levels (e. g. PALPA [10]) that were available so far, are time-consuming and therefore not suitable for use in the early phase of stroke. The ScreeLing consists of three subtests, each assessing processing at one linguistic level: Semantics, Phonology, Syntax. These subtests were validated against the judgment of an experienced linguist with respect to the presence or absence of a semantic, phonological and/or syntactic deficit. Semantics and Phonology point out to have a good diagnostic accuracy. At the optimal cut-off point (i. e. maximizing the sum of sensitivity and specificity), specificity of these subtests is perfect, but sensitivity is relatively low. The best cut-off point for clinical use may differ from the 'optimal' cut-off point, for example if sensitivity is judged to be more important than specificity. For Semantics all task-types (see description in the Appendix) contributed to the diagnostic accuracy (errorrates varied from 12 to $35 \%$ ). For Phonology, mainly Phon 1 (repetition) and Phon 2 (reading aloud) were responsible for predicting the presence of a phonological deficit (error rates: 18 and 35\%), whereas Phon 3 (reading backwards) and Phon 4 (auditory lexical decision) turned out not to be very useful (error-rates respectively $65 \%$ and $59 \%$ ). The subtest for syntax is less accurate; some patients who have a syntactic deficit according to the linguist, score above cut-off on this subtest. Error- analysis is needed to obtain more insight into the reasons for discrepancy between the results of the ScreeLing and the judgment of the linguist. A first analysis revealed that for only one task-type within Syntax, Syn 4 (repetition of sentences), the error-rate was low enough $(24 \%)$ to be useful for predicting a syntactic deficit; the other question-types had an error-rate of $41 \%$.

The mean Phonology score was higher than the mean Semantics score and the mean Syntax score. It is possible that Phonology was easier than Semantics and Syntax or less strenuous to patients. Alternatively, phonological deficits may be more rare than semantic and syntactic deficits. At present, data about the frequency of occurrence of linguistic disorders in an early stage of aphasia are lacking. From our patients' subtest scores, we conclude that the performance on the three subtests was diverse, which shows that selective linguistic disorders may be detected already in the acute phase. This finding is in line with a study by Van Zandvoort et al. [18], showing that neuropsychological screening in the acute phase of stroke may reveal specific disorders.

In conclusion, the ScreeLing is an accurate test for detecting aphasia between 2 and 11 days after stroke. Unlike other screening-tests, the ScreeLing is suitable for revealing deficits at the main linguistic levels, especially semantic and phonological deficits. This is important for guiding early aphasia therapy, the importance of which is increasingly recognized $[12,13]$. For the remedying of each of these deficits, well-evaluated therapeutic tools are available $[14,16,19]$. Knowledge of the degree and rate of recovery of each linguistic deficit will affect early therapeutic decisions with respect to which linguistic deficit should be treated and when. In a follow-up study, we are currently investigating the degree and rate of recovery of linguistic deficits from the acute phase of aphasia until 6 months post onset. Subsequently, item analysis will be performed and the ScreeLing will be adjusted to increase diagnostic accuracy.

\footnotetext{
Acknowledgments This study was supported by NWO-Chronic Diseases, nr. 94033008. The authors thank the patients who participated in the study. Furthermore, we are grateful to Miranda van Rijn, speech language therapist, the neurologists of the Erasmus Medical Center and the neurologists and the speech language therapist, Yvonne Worms, of the Medical Center Rijnmond Zuid for their contributions to the study.
}

\section{References}

1. Al-Khawaja I, Wade DT, Collin CF (1996) Bedside screening for aphasia: a comparison of two methods. J Neurol 243:201-204

2. Boller F, Dennis M (1979) Auditory comprehension: clinical and experimental studies with the Token Test. Academic Press Inc, New York
3. Cicerone KD, Dahlberg C, Kalmar K, Langenbahn DM, Malec JF, Bergquist TF, Felicetti T, Giacino JT, Harley JP, Harrington DE, Herzog J, Kneipp S, Laatsch L, Morse PA (2000) Evidencebased cognitive rehabilitation: recommendations for clinical practice. Arch Phys Med Rehabil 81:1596-1615
4. Crary MA, Haak NJ, Malinsky AE (1989) Preliminary psychometric evaluation of an acute aphasia screening protocol. Aphasiology 3:611-618

5. Code C (2001) Multifactorial processes in recovery from aphasia: developing the foundations for a multileveled frameword. Brain Lang 77:25-44 
6. De Renzi E, Faglioni P (1978) Normative data and screening power of a shortened version of the Token Test. Cortex 14:41-49

7. De Renzi E, Vignolo LA (1962) The Token Test: a sensitive test to detect receptive disturbances in aphasics. Brain 85:665-678

8. Enderby PM, Wood VA, Wade DT, Hewer RL (1987) The Frenchay Aphasia Screening Test: a short, simple test for aphasia appropriate for non-specialists. Int Rehabil Med 8:166-170

9. Howard D, Patterson K (1989) Models for therapy. In: Seron X, Deloche G (eds) Cognitive approaches in neuropsychological rehabilitation. Lawrence Erlbaum, London-Hillsdale N.J, pp 39-62

10. Kay J, Lesser R, Coltheart M (1992) Psycholinguistic Assessment of Language Processing in Aphasia (PALPA). Lawrence Erlbaum Associates, London

11. Laska AC, Hellblom V, Murray V, Kahan T, Von Arbin M (2001) Aphasia in acute stroke and relation to outcome. Intern Med 249:413-422
12. Robey RR, Schultz MC (1998) A model for conducting clinical outcome research: an adaptation of the standard protocol for use in aphasiology. Aphasiology 12:787-810

13. Robertson IH, Murre JMJ (1999) Rehabilitation of Brain Damage: brain plasticity and principles of guided recovery. Psychol Bull 125:544-575

14. Robson J, Marshall J, Pring T, Chiat S (1998) Phonological naming therapy in jargon aphasia: positive but paradoxical effects. J Int Neuropsychol Soc 4:675-686

15. Thomessen B, Thoresen G, BautzHolter E, Laake K (1999) Screening by nurses for aphasia in stroke: the Ullevaal Aphasia Screening (UAS) test. Disabil Rehabil 21:110-115

16. Thompson CK, Shapiro LP, Roberts MM (1993) Treatment of sentence production deficits in aphasia: a linguistic specific approach to wh-interrogative training and generalization. Aphasiology 7:111-133
17. Van Harskamp F, Van Dongen HR (1977) Construction and validation of different short forms of the Token Test. Neuropsychologia 15:467-470

18. Van Zandvoort MJE (2001) Cognitive function following single lacunar infarct (dissertation). Offsetdrukkerij Ridderprint BV

19. Visch-Brink EG, Bajema IM, van de Sandt-Koenderman ME (1997) Lexical semantic therapy: BOX. Aphasiology 11:1057-1115

20. Visch-Brink EG, Van de Sandt-Koenderman WME (2003) ScreeLing: een linguïstische screeningstest voor afatische patiënten. Stem-, Spraak- en Taalpathologie (in press) 